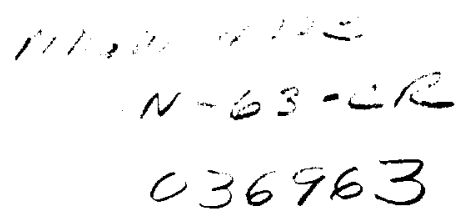

\title{
Design of a Minimum Surface-Effect Tendon-Based Microactuator for Micromanipulation
}

NASA-CR-205001

\author{
Michael Goldfarb \\ James H. Lipsey \\ Department of Mechanical Engineering \\ Vandertilt University \\ Nashville, TN 37235
}

\begin{abstract}
A piezoelectric ( $P Z T$ ) stack-based actuator was developed to provide a means of actuation with dynamic characteristics appropriate for small-scale manipulation. In particular, the design incorporates a highly nonlinear. large-ratio transmission that provides approximately two orders of magnitude motion amplification from the PZT stack. In addition to motion amplification, the nonlinear transmission was designed via optimization methods to distort the highly non-uniform properties of a piezoelectric actuator so that the achievable actuation force is nearly constant throughout the actuator workspace. The package also includes sensors that independently measure actuator output force and displacement, so that a manipulator structure need not incorporate sensors nor the associated wires. Specifically, the actuator was designed to output a maximum force of at least one Newton through a stroke of at least one millimeter. For purposes of small-scale precision position and/or force control, the actuator/sensor package was designed to eliminate stick-slip friction and backlash. The overall dimensions of the actuator/sensor package are approximately $40 \times 65 \times 25 \mathrm{~mm}$.
\end{abstract}

\section{Introduction}

Small scale manipulator design, and in particular design for the purpose of human-controlled teleoperation, is severely restricted by the lack of available actuator technology. Smaller versions of conventional scale actuators, such as DC motors, are typically not appropriate for micromanipulator actuation, due in part to unfavorable power scaling and in part to the increased significance of stick-slip forces on small scales and the control difficulties associated with this type of friction $[1,4]$. To avoid the control problems associated with the significant influence of small scale friction, researchers have investigated several deformation-based or non-contact candidate actuators for micromanipulation, including piezoelectric ceramic, shape memory alloy, magnetostrictive alloys, electrostrictive alloys, electrostatic actuation, and electromagnetic actuation [1,4]. Despite these several possibilities, there remains a significant void in micromanipulator actuator candidates. Each actuator seems to lack some critical feature, such as open-loop stability and/or the required bandwidth, power, range of motion, or force.

This paper describes an actuator package that was designed to fill the microactuator void. The proposed actuator package, which is based around a PZT stack actuator, provides a bandwidth on the order of several hundred Hertz, exhibits a roughly uniform force in excess of one Newton over a linear stroke of one millimeter, does not exhibit any stick-slip friction or backlash, and incorporates sensors that measure both actuator position and force.

\subsection{Manipulator Specifications}

The tendon-based microactuator was designed specifically to actuate a teleoperated micromanipulator, and as such most of the associated design requirements are derived from the manipulator specifications. The micromanipulator was designed for use under an optical stereomicroscope, and thus for a workspace and resolution of approximately $125 \mathrm{~mm}^{3}$ (a cube $5 \mathrm{~mm}$ on a side) and $1 \mu \mathrm{m}$, respectively. The latter is a function of the wavelength of light. Such scaling would entail a geometric scaling factor of approximately $\mathbf{3 0}$ for typical human dexterous manipulation. Since human-controlled telemanipulation is a targeted mode of operation, the manipulator was designed for teleoperative transparency, which generally requires position and force bandwidths on the order of 15 and $300 \mathrm{Hertz}$, respectively [2]. Also, as previously discussed, the manipulator, actuator, and transmission were designed to minimize surface-effects 
and thus enable precision position and/or force control at a small scale.

A simple analysis of the mechanics of scaling offers insight into actuator force requirements. The types of forces associated with system mechanics can be associated with one of three generalized energetic behaviors: forces associated with displacement (generalized stiffness), forces associated with velocity (generalized damping), and forces associated with acceleration (generalized inertia). Simple analyses show that for kinematic and geometric similarity characterized by the scaling factor $n$, the forces associated with a generalized stiffness scale as $n^{2}$, those associated with damping as $n^{3}$, and those associated with inertia as $n^{4}$. Designing for the forces associated with a generalized stiffness therefore provides what is likely the most conservative estimate of required force. Since the manipulator was designed for a nominal geometric scaling factor of 30 , scaling 50 Newtons (a rather large force for human dexterous manipulation) to the micromanipulator would require an equivalent endpoint force of approximately 50 milliNewtons.

\subsection{Actuator Specifications}

The actuators were designed for a tendon-based configuration. This configuration enables relatively remote location of the actuator packages and also provides the option of additional motion amplification, thus alleviating size constraints and reducing the requisite actuator amplification. Since the tendon configuration can easily provide a five-fold motion amplification, the actuator need only provide 250 milliNewtons of force through a $1 \mathrm{~mm}$ output stroke to provide the requisite manipulator specifications. Since the manipulator joints were designed as flexures and require force to overcome their inherent stiffness, the actuator was designed to produce approximately 1 Newton of force over the $1 \mathrm{~mm}$ stroke. The package was also designed to include sensors that independently measure actuator output force and tendon displacement, so that the manipulator structure need not incorporate sensors nor the associated wires.

\section{A Piezoelectric Stack-Based Design}

Of the design requirements described above, power, bandwidth, and resolution are fundamental, meaning these requirements must be displayed by the power transducer and deficiencies cannot be compensated for by any sort of transmission. Inadequate force or displacement can be corrected through the use of a suitably designed transmission. Additionally, for purposes of precision position and/or force control, an appropriate power transducer should be absent of backlash and stick-slip friction. A review of candidate actuators indicates that piezoelectric ceramic is a good candidate for providing the desired actuation performance $[1,3]$.

A typical lead-zirconate-titanate (PZT) piezoelectric actuator can perform step movements with a resolution on the order of one in 10,000 . These actuators offer openloop stable operation with the power and bandwidth necessary for the specified motion. The primary inadequacy of the PZT as a microrobotic actuator is that the strain-based deformations that it provides are limited to approximately $0.1 \%$. A piezoelectric stack that could provide the desired displacement of $1 \mathrm{~mm}$ in a directdrive fashion would therefore have to be approximately one meter in length, an obviously unreasonable geometry. A typical piezoelectric stack actuator, such as the stack incorporated in the microactuator design (Tokin model \#AE0505D16), is twenty millimeters in length and supplies approximately 15 microns of unloaded displacement. Aside from the limited displacement, the other major limitation of a PZT stack actuator is that the output force is highly non-uniform through the actuator stroke, and in fact decreases linearly with increasing stroke. The maximum force versus displacement for the PZT stack incorporated into the microactuator is shown in Figure 1. Incorporating a PZT stack to achieve the desired microactuator performance therefore requires a large-ratio, nonlinear transmission that will distort the direct-drive properties of the PZT to obtain a near-constant output force with a net displacement amplification of approximately one hundred times. As previously discussed, precision position and force control require that the open loop behavior of the manipulator, which includes the actuator and transmission, be free of backlash and stickslip friction. Note also that the choice of tendon actuation does not unnecessarily constrain the output properties of the PZT for purposes of a force-reflective or forcecontrolled manipulator. PZT stack actuators provide controllable force only in compression. Bidirectional force control thus requires two PZT actuators per axis. A tendon configuration simply converts the normally compressive mode of operation to a structurally more stable tensile mode.

\section{Design for Uniform Force Characteristics}

As previously stated, the output force characteristics of a PZT stack are highly non-uniform, as depicted in Figure 1. Converting these characteristics to uniform microactuator properties of the specified magnitudes would require a nonlinear transmission of the form: 


$$
\frac{F_{\text {mut }}}{F_{\text {in }}}=\frac{F_{\text {max }}}{F_{o}-k x_{p}}
$$

where $F_{\max }$ is the maximum actuator output force (1 Newton in this case), $F_{o}$ is the zero-displacement PZT stack force, $k$ is the rate at which PZT force decreases with increased displacement ( $53.3 \mathrm{Newtons} /$ micron for the PZT of Figure 1), and $x_{p}$ is the displacement of the PZT stack. Employing variational principles and assuming no energy is stored or dissipated in the transmission, the corresponding kinematic relationship would be:

$$
x_{\text {out }}=\frac{F_{o} x_{p}-\frac{k}{2} x_{p}^{2}}{F_{\max }}
$$

where $x_{\text {out }}$ is the output displacement of the microactuator package and the other variables are as defined previously. Graphical representation of both the required force transmission and the desired kinematics are shown in Figure 2.

One convenient and compact means of obtaining kinematics similar to those just described is by incorporating an isosceles linkage in a compressive mode, as shown in Figure 3. This type of linkage is kinematically characterized by the following relationship:

$$
x_{\text {out }}=\sqrt{l^{2}-\left(l \cos \theta_{o}-y_{\text {in }}\right)^{2}}-l \sin \theta_{o}
$$

where the variables are as defined in Figure 3. Proper choice of the link length $l$ and the initial angle $\theta_{o}$ can closely approximate the desired relationship of the ideal kinematics, as shown in Figure 4. Note that the isosceles linkage must act in compression, i.e.: the angle must increase with the input displacement. A similar linkage operating in the inverse mode would further exaggerate the highly non-uniform properties of the PZT.

\subsection{Design Morphology}

A combination of constraints determines the fundamental configuration of the transmission. First, the PZT outputs a forced elongation; second, the isosceles linkage must incorporate an input of forced compression; and third, the desired microactuator output is one of forced shortening. These constraints determine that the transmission consist of three stages: a first stage that converts PZT lengthening to shortening, a second stage that incorporates isosceles geometry to favorably distort the force

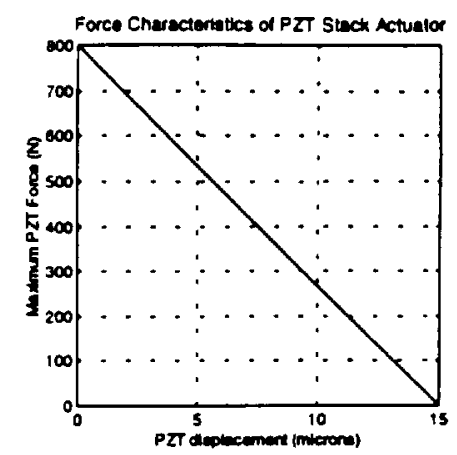

Figure 1. Force versus displacement envelope for PZT stack actuator (Tokin model \#AE0505D16).
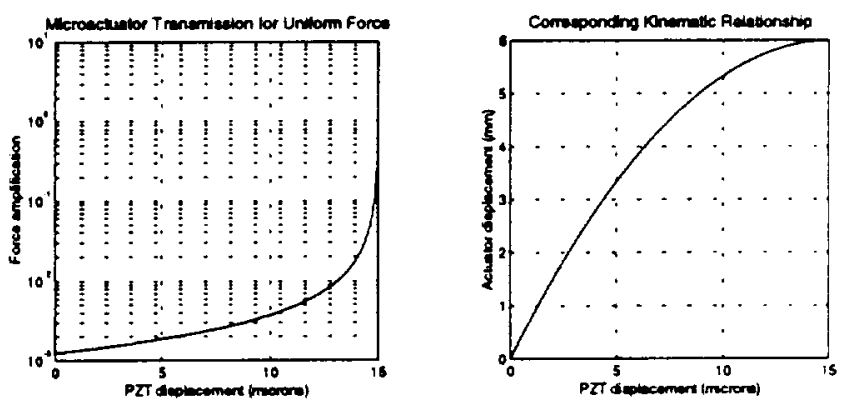

Figure 2. Characteristics of ideal force transmission (left) and corresponding kinematic behavior (right).

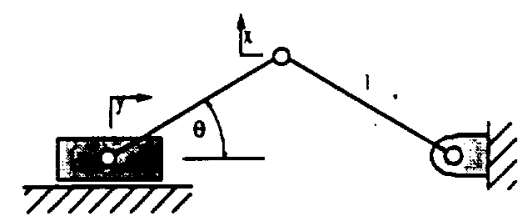

Figure 3. Schematic representation of isosceles linkage.

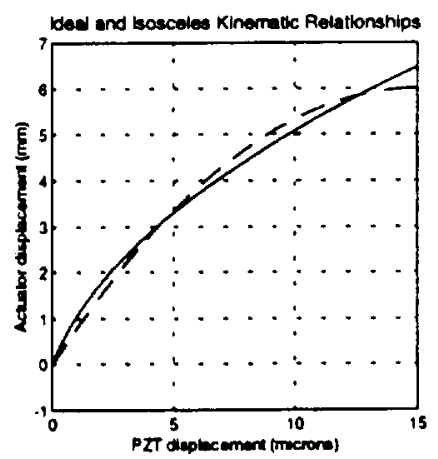

Figure 4. Kinematic characteristics of ideal transmission (dashed) and isosceles transmission (solid). 
characteristics, and a third stage that converts the lengthening output of the second stage to the desired shortening output for use with a tendon configuration.

In addition to appropriate kinematics, the actuator transmission must have a sufficiently large output stiffness. In other words, the transmission must be capable of maintaining the prescribed input-output kinematic relationship while transmitting a significant load. This is particularly important for the design of a manipulator, which unlike a micropositioner, generally involves significant forces of interaction. A large output stiffness is of particular importance in the design of the first stage, which must transmit on the order of hundreds of Newtons through a displacements on the order of microns. Unless the first stage has a stiffness on the order of $1 \mathrm{~N} / \mu \mathrm{m}$, it will, under load, effectively lose the entire displacement of the PZT in structural deformation. Since the magnitude of the required stiffness decreases with the square of the transmission ratio of each stage, maintaining such a large output stiffness is significantly less of an issue with the second and third stages. The first stage must therefore be designed with a geometry that is conducive to a large output stiffness. The two primary configurations for kinematic amplification are the previously discussed isosceles configuration and the more common lever configuration. The output stiffness of a conventional lever-type linkage is essentially the bending stiffness of the link, given by:

$$
k_{l}=\frac{E b h^{3}}{4 l^{3}}
$$

where $E$ is the modulus of elasticity, $b$ and $h$ are the link cross-sectional width and height, respectively, and $l$ is the link length. The (locally linear) stiffness of an isosceles linkage is given by:

$$
k_{i}=\frac{2 E b h}{l} \sin ^{2} \theta
$$

where $l$ is the length of each link and $\theta$ is the isosceles angle. Since a large transmission requires a small angle $\theta$, the isosceles stiffness generally tends to be inadequate. Additionally, the stiffness is only affected linearly by all aspects of link geometry. In contrast, the fact that beam height affects lever stiffness in a cubic manner affords significant stiffness for beams that incorporate an aspect ratio $h / l \approx l$. This analysis, supported by results of finite clement analysis, indicates that the first stage should be of a conventional lever type configuration. As previously mentioned, the second stage was designed in a compres- sive isosceles configuration to provide favorable distortion for uniform force properties. The third stage, which functions primarily as the sensing stage, is also an isosceles linkage.

\subsection{Flexure-Based Revolute Joints}

The combined stages of the transmission utilize twenty flexure-based revolute joints. Flexure-based joints enable angular motion without the backlash or stick-slip friction that would be imminently present in a small-scale rolling or sliding contact bearing, and thus eliminate potentially intractable control difficulties. The microactuator transmission utilizes two types of flexure-based joints: conventional flexures and split-tube flexures. Conventional flexure hinges rely on the bending of a short, thin section of material that is significantly more compliant than the members it connects. The split-tube flexures, which are described in detail in a concurrent paper, provide significantly better revolute properties than conventional flexures without the backlash and stick-slip friction of conventional revolute joints. These flexures are formed by removing a slender section from a thin-wall hollow tube. Removal of the section provides for compliance along the axis opposite the removed section, while preserving stiffness in other directions of rotation and in tension and compression. Conventional flexures are utilized in the first and third stages where the joint motions are small and the loading primarily planar. Since the second stage exhibits relatively large joint angles and is subjected to significant out-of-plane loading, split-tube flexures are used.

\section{Design Optimization}

As previously mentioned, the micromanipulator specifications require an actuator output of at least 1 Newton over a stroke of at least $1 \mathrm{~mm}$. As illustrated by Figure 4, an appropriately designed isosceles linkage can closely approximate the behavior required to achieve this output. Because the actuator involves three stages, appropriate choice of linkage geometry becomes significantly more complex. The desired output behavior can be described in a single degree-of-freedom; therefore, the set of geometric variables involved in the design of the transmission were incorporated into a single cost function to enable optimization of the microactuator design.

The initial optimization utilized a cost function that incorporated the kinematic behavior illustrated by Figure 4. Employing variational mechanics, the input-output force relationship can be represented by a single degreeof-freedom relationship that involves two independent 
parameters. For the linkage of Figure 3, if the single degree-of-freedom is $y$ and the two independent parameters are $l$ and $\theta$, this relationship is given by:

$$
F_{\text {uut }}=\frac{\sqrt{l^{2}-\left(l \cos \theta_{o}-y\right)^{2}}}{\left(l \cos \theta_{o}-y\right)} F_{\text {in }}
$$

Using $F_{\text {in }}$ and $y$ as boundary conditions, a cost function can be constructed by taking difference between $F_{\text {out }}$ and a desired output force:

$$
J=\int_{0}^{y_{\max }}\left|F_{\text {desire }}-\frac{\sqrt{l^{2}-\left(l \cos \theta_{o}-y\right)^{2}}}{\left(l \cos \theta_{o}-y\right)} F_{\text {in }}(y)\right| d y
$$

The transmission design is optimized by utilizing the PZT characteristics of Figure 1 as the input to the first stage and modeling the output of each stage as input to the next. Finally, the collective cost function is minimized by manipulating the geometric constraints.

The force relationships of this optimization, however, were derived under the assumption that no significant energy is stored in the transmission structure. Given that the structure incorporates flexure-based revolute joints, this is a rather poor assumption. The transmission output force is more accurately modeled as the force that remains after energy is stored deforming the flexures. A more accurate input-output force relationship was derived by assuming each joint exhibits a linear stiffness, as shown in Figure 5. Note also that the isosceles linkage of Figure 5 includes four rather than three revolute joints. This configuration is necessitated by implementation, and an assumption of symmetry is made to void the additional degree-of-freedom. For the linkage of Figure 5, the output force relationship is given by:

$$
\begin{aligned}
F_{\text {lut }}=\frac{1}{\left(l \cos \theta_{i j}-y\right)}[ & 4 k\left(\theta_{o}-\cos ^{-1}\left(\frac{l \cos \theta_{a}-y}{l}\right)\right) \\
& \left.-F_{i n}(y) \sqrt{l^{2}-\left(l \cos \theta_{o}-y\right)^{2}}\right]
\end{aligned}
$$

and the cost function is adjusted accordingly.

\section{Microactuator Design}

Figure 6 shows the optimized force output characteristics of the microactuator, given the input characteristics

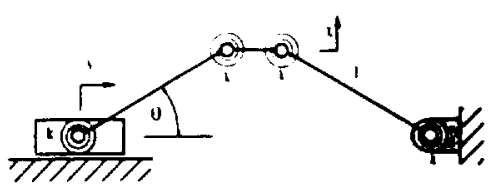

Figure 5. Model of isosceles linkage as implemented. including tlexure joint stiffnesses.

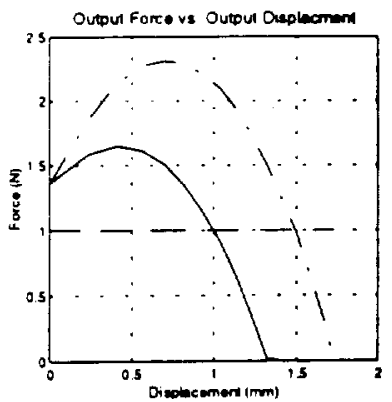

Figure 6. Optimized output characteristics of actuator showing desired (dashed), kinematically optimized (dash-dot), and energetically optimized (solid) characteristics.

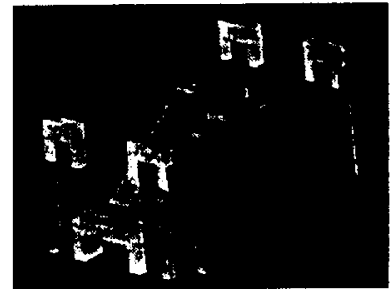

Figure 7. Solid model of microactuator package.

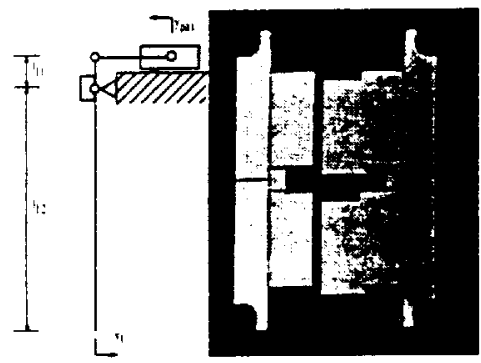

Figure 8. Quarter model and solid model of the first stage.

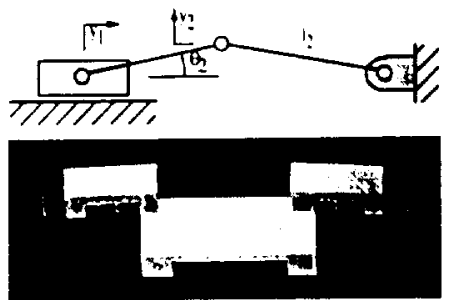

Figure 9. Kincmatic model and solid model of the second stage. 


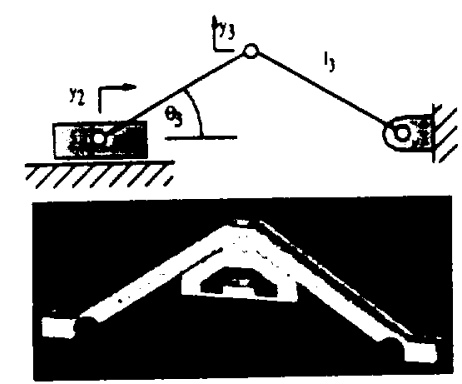

Figure 10. Kinematic model and solid model of the third stage.

of the PZT shown in Figure 1. A solid model of the resulting microactuator package is shown in Figure 7.

A solid model and corresponding kinematic model of the first stage are shown in Figure 8. To maximize the output stiffness, the stage is constructed of steel alloy, and fabricated using a wire electrical discharge machine (EDM) process. The resulting geometry, defined in Figure 8 , incorporates beam lengths of $l_{11}=1.5 \mathrm{~mm}$ and $l_{12}=27 \mathrm{~mm}$, providing a roughly constant displacement amplification of 18 . Finite element analysis of the first stage confirms that, in an unloaded condition, $135 \mathrm{mi}$ crons of displacement are produced at each output location from a 15 micron input generated by the PZT. FEM analysis also indicates that the first stage exhibits an output stiffness of roughly $1 \mathrm{~N} / \mu \mathrm{m}$, indicating the stage will hold kinematically true while under maximum load.

A solid model of the second stage, along with a corresponding kinematic model, are shown in Figure 9. The geometry of the second stage, as defined in the figure, incorporates link lengths and an initial angle of $l_{2}=7.5 \mathrm{~mm}$ and $\theta_{2 o}=2.5^{\circ}$, respectively. At maximum PZT displacement, $\theta_{2}$ increases to $11.2^{\circ}$. The net change in angle is therefore approximately $8^{\circ}$, through which the linear displacement amplification ranges from 22.9 to 5.1 .

The third stage is shown in Figure 10. The geometry of the third stage, as defined in the figure, incorporates link lengths and an initial angle of $l_{5}=30 \mathrm{~mm}$ and $\theta_{30}=35^{\circ}$, respectively. At maximum PZT displacement, $\theta_{3}$ decreases to approximately $31^{\circ}$, resulting in a net change in angle of $4^{\circ}$ and a corresponding change in linear displacement amplification from 1.4 to 1.6. Since the required output stiffness of the third stage is small and operation is best served with compliant flexures, the third stage is designed of aluminum alloy, which can be fabricated utilizing a wire electrical discharge machine process. An important function of the third stage is strain based sensing of position and force, as indicated in Figure 10. Position is measured by strain gages mounted on the lower flexure hinges, and force by strain gages mounted on a load cell. The load cell was designed to provide sufficient strain for measurement while minimizing the structural deformation, thus avoiding lost motion. The design affords on the order of 1000 microstrain under full load while losing less than $1 \%$ of the actuator motion. The position-based strain similarly provides more than 1000 microstrain under full displacement. An FEM analyses of the structure under full displacement, and a second analyses of the stage under full load indicate less than $1 \%$ crosstalk between the position and force signals.

\section{Conclusion}

The authors developed a minimum surface-effect design for a tendon-based microactuator, intended for use in a teleoperated micromanipulator. The microactuator package is designed to output a near-constant force of one Newton over a one millimeter range of motion. Sensors incorporated in the design provide the package with integral force and position sensing capability. A prototype of the design presented in this paper is currently under construction; once functional, the system performance will be characterized and subsequently presented in a future publication.

\section{Acknowledgments}

Support for this work was provided by NASA Grant No. NAGW-4723. The authors gratefully acknowledge this support.

\section{References}

[1] Dario, P., Valleggi, R., Carrozza, M.C. Montesi, M.C., and Cocco, M. "Microactuators for Microrobots: A Critical Survey." Joumal of Micromechanics and Microengineering, vol. 2, pp. 141-157, 1992.

[2] Fischer P., Daniel R., and Siva K.V., "Specification and Design of Input Devices for Teleoperation," Proceedings of the IEEE Conference on Robotics and Automation, pp. 540-545, 1990.

[3] Goldfarb, M., and Celanovic, N. "Behavorial Implications of Piezoelectric Stack Actuators for Control of Micromanipulation." Proceedings of the IEEE Conference on Robotics and Automation, April, 1996.

[4] Trimmer, W. and Jebens, R., "Actuators for Micro Robots," In Proceedings of the IEEE International Conference on Robotics and Automation, pp. 1547. 1552. 1989. 